Supporting Information for

\title{
Predicting Molecular Fingerprint from Electron-Ionization Mass Spectrum with Deep Neural Networks
}

\author{
Hongchao Ji, Hanzi Deng, Hongmei Lu*, Zhimin Zhang* \\ College of Chemistry and Chemical Engineering, Central South University, Changsha 410083, PR China. \\ Corresponding Author E-mail address: hongmeilu@csu.edu.cn; zhangzhimin@csu.edu.cn
}

Table S2. Parameters comparison of fingerprints' prediction for XGBoost method

\begin{tabular}{|c|c|c|c|c|c|}
\hline FP Index & Parameters & Accuracy & Precision & Recall & F1 \\
\hline 12 & max_depth $=2, \mathrm{n}$ _estimators $=50$ & 0.830 & 0.642 & 0.231 & 0.340 \\
\hline 12 & max_depth $=2, \mathrm{n}$ _estimators $=70$ & 0.835 & 0.657 & 0.282 & 0.395 \\
\hline 12 & $\max \_$depth $=2, \mathrm{n}$ _estimators $=100$ & 0.846 & 0.688 & 0.333 & 0.448 \\
\hline 12 & max_depth $=3, \mathrm{n} \_$estimators $=50$ & 0.837 & 0.673 & 0.310 & 0.424 \\
\hline 12 & max_depth $=3, \mathrm{n} \_$estimators $=70$ & 0.849 & 0.711 & 0.358 & 0.476 \\
\hline 12 & max_depth $=3$, n_estimators $=100$ & 0.857 & 0.734 & 0.398 & 0.516 \\
\hline 12 & max_depth $=4, \mathrm{n}$ _estimators $=50$ & 0.848 & 0.705 & 0.362 & 0.478 \\
\hline 12 & max_depth $=4, \mathrm{n} \_$estimators $=70$ & 0.856 & 0.725 & 0.402 & 0.517 \\
\hline 12 & max_depth $=4, \mathrm{n}$ _estimators $=100$ & 0.859 & 0.709 & 0.444 & 0.546 \\
\hline 271 & max_depth $=2, \mathrm{n}$ _estimators $=50$ & 0.814 & 0.805 & 0.847 & 0.826 \\
\hline 271 & max_depth $=2, \mathrm{n}$ _estimators $=70$ & 0.817 & 0.814 & 0.847 & 0.831 \\
\hline 271 & max_depth $=2, \mathrm{n}$ _estimators $=100$ & 0.827 & 0.821 & 0.861 & 0.840 \\
\hline 271 & max_depth $=3$, n_estimators $=50$ & 0.831 & 0.814 & 0.875 & 0.843 \\
\hline 271 & max_depth $=3, \mathrm{n}$ _estimators $=70$ & 0.835 & 0.823 & 0.874 & 0.848 \\
\hline 271 & max_depth $=3$, n_estimators $=100$ & 0.848 & 0.840 & 0.880 & 0.859 \\
\hline 271 & max_depth $=4, \mathrm{n} \_$estimators $=50$ & 0.845 & 0.827 & 0.890 & 0.857 \\
\hline 271 & max_depth $=4$, n_estimators $=70$ & 0.851 & 0.837 & 0.891 & 0.863 \\
\hline 271 & max_depth $=4$, n_estimators $=100$ & 0.862 & 0.846 & 0.900 & 0.873 \\
\hline
\end{tabular}




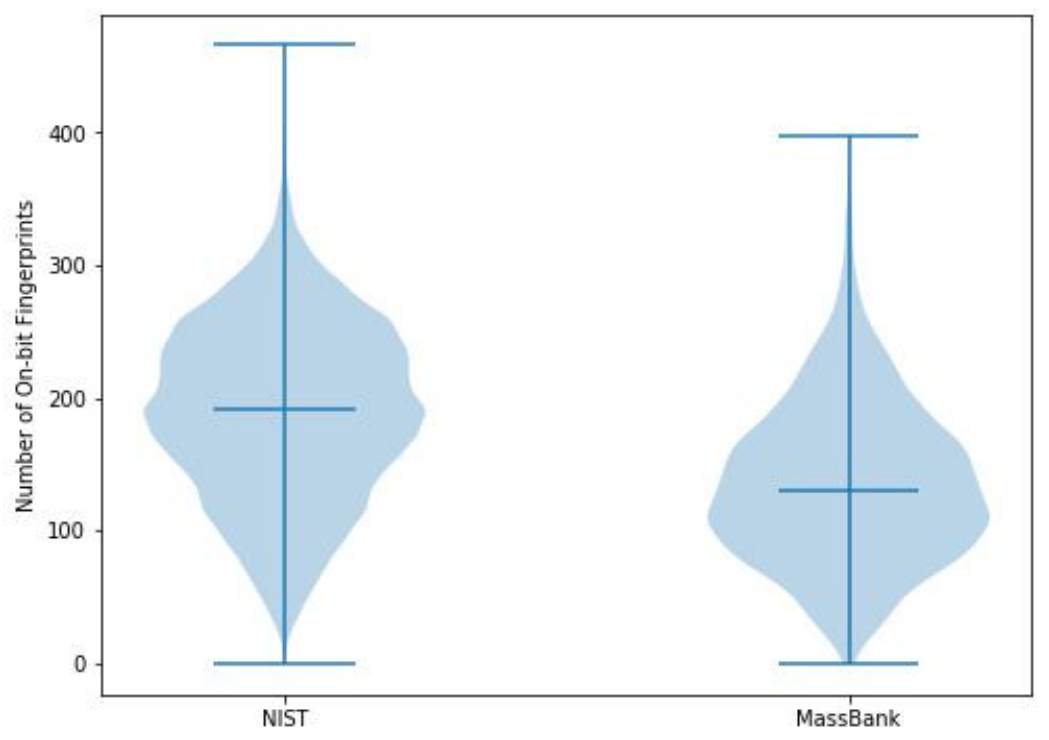

Fig S1. The number of on-bit fingerprints of NIST and MassBank.
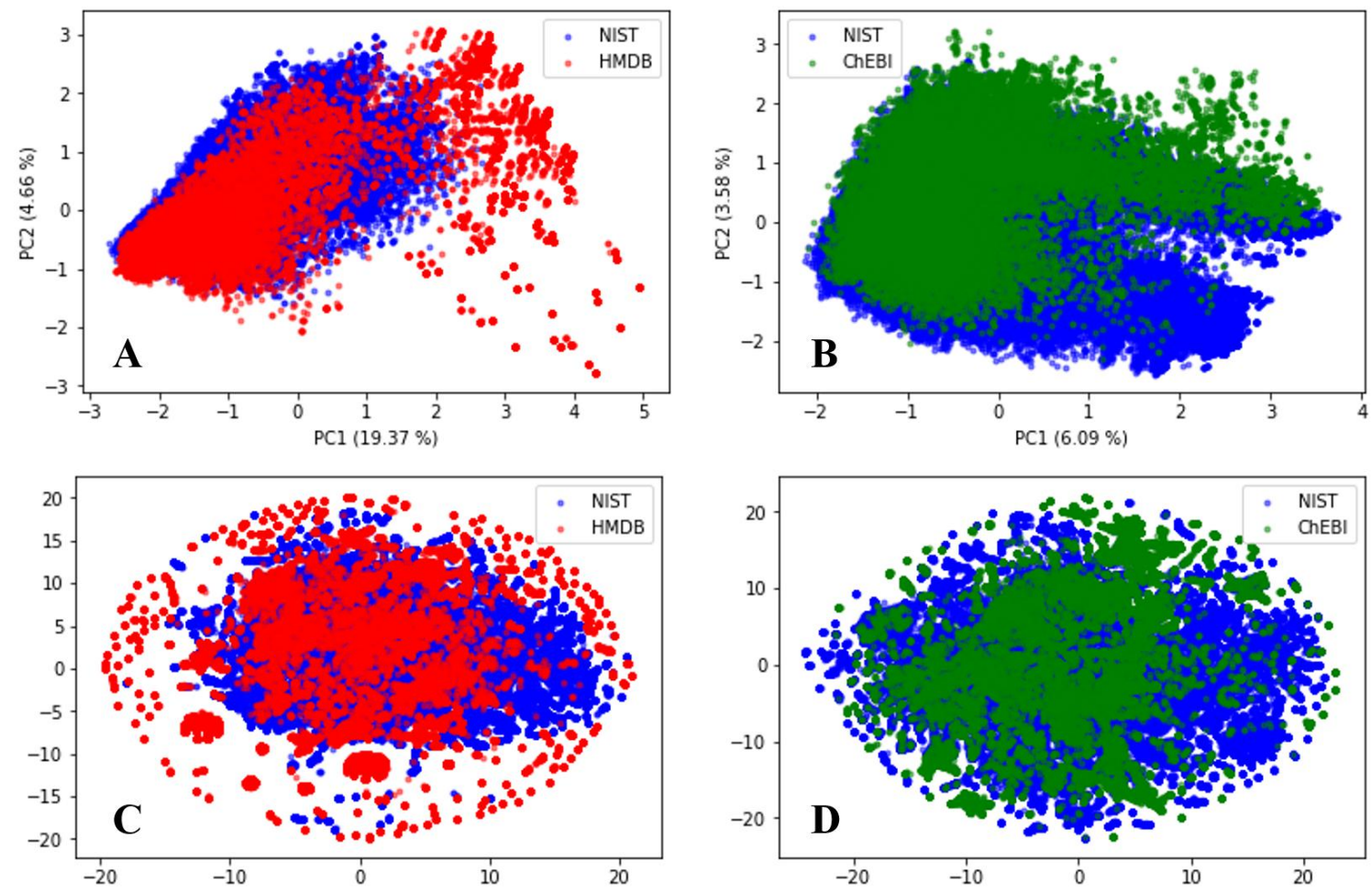

Fig S2. The chemical space comparison of NIST, HMDB and ChEBI compounds. A and B are the visualization with PCA. C and D are the visualization with T-SNE. 

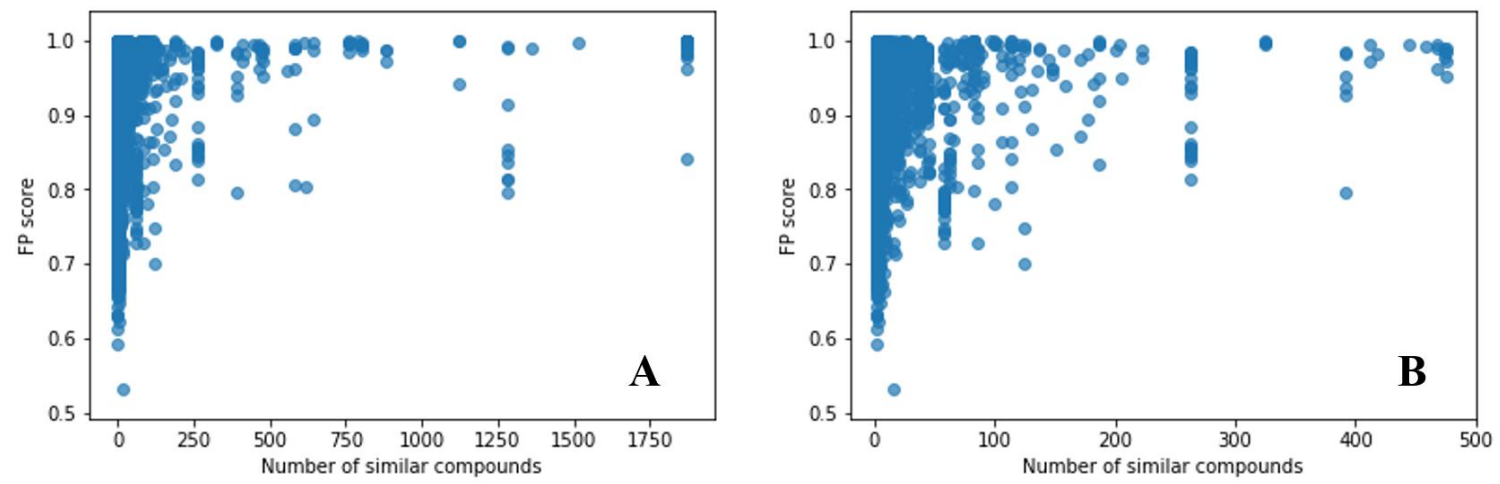

Fig S3. Relationship between Jaccard similarity and predicted FP score. The $\mathrm{x}$-axis shows the number of molecules in the training set which have a Dice similarity of greater than 0.8 to the query molecule. The y-axis shows the FP score between the predicted spectra and the main library spectra for the query molecule.
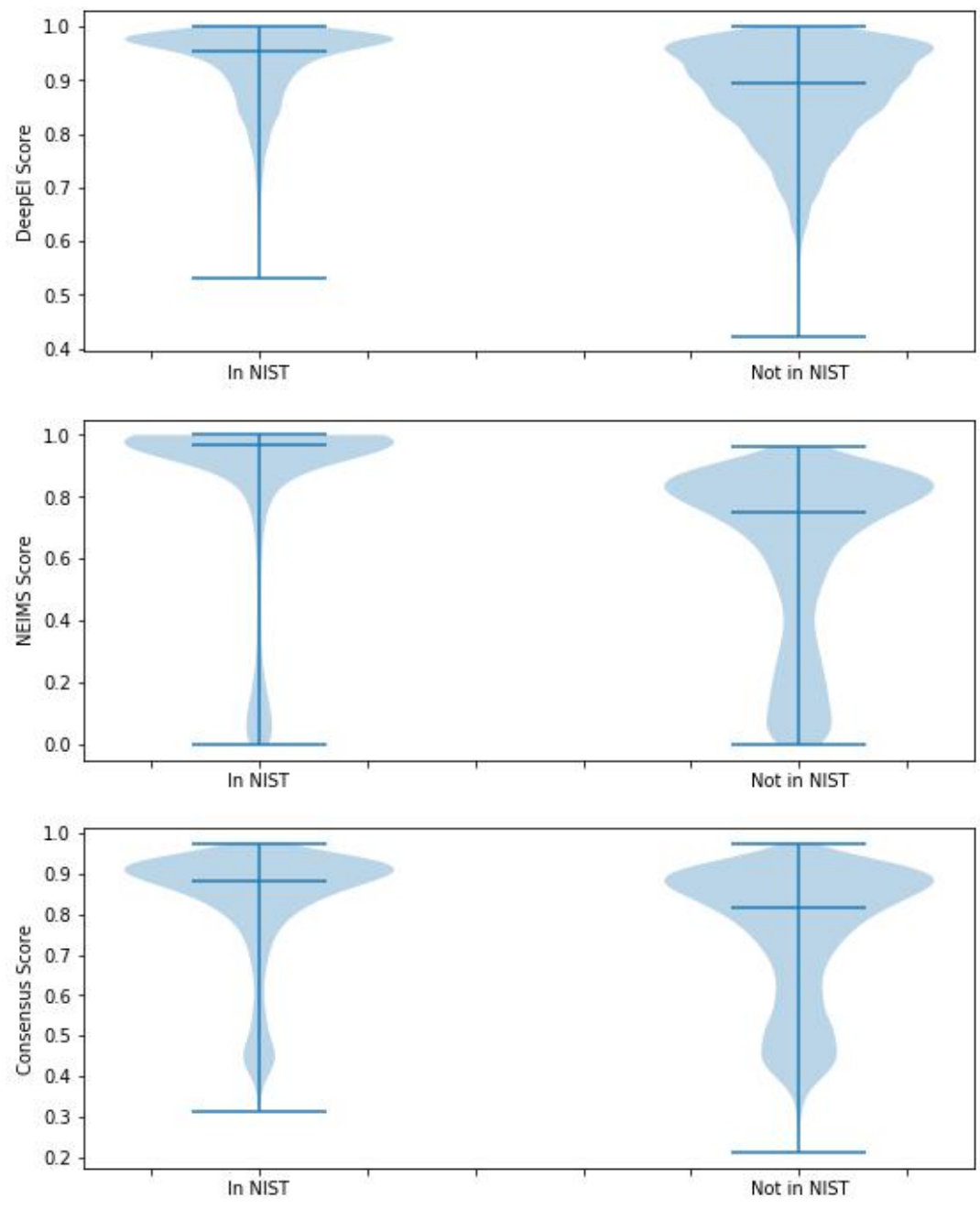

Fig S4. Distribution of scores of the true structures given by DeepEI, NEIMS and their combination. 